\section{Impact of obesity on childhood kidney}

\author{
Kazunari Kaneko, ${ }^{1}$ Takahisa Kimata, ${ }^{1}$ \\ Shoji Tsuji, ${ }^{1}$ Kazumi Shiraishi, ${ }^{2}$ \\ Kuniaki Yamauchi,2 Mutsumi Murakami,2 \\ Teruo Kitagawa² \\ 1Department of Pediatrics, Kansai \\ Medical University; ${ }^{2}$ Tokyo Health Service \\ Association, Japan
}

\section{Abstract}

Obese patients are known to have greater risks to develop hypertension, coronary vascular disease, and insulin resistance, and more attention has been recently paid to the impact of obesity on kidney. This study was conducted to investigate whether obese children have higher risk of renal injury as well as adults. Eighteen hundred and thirty school children aged 6-14 years with abnormal urinary findings on thrice occasions detected by the screening program for renal disease in Japan were enrolled. Of them, 27 children with nephritis or suspected nephritis diagnosed by persistent proteinuria with hematuria were compared to 588 without urinary abnormalities regarding their body mass index (BMI), blood pressure (BP), and serum level of total cholesterol. BMI and systolic BP (mmHg) were significantly higher in the former than in the latter. As a result, obesity may be associated with the development of renal injury even in childhood.

\section{Introduction}

It is well documented that obese patients are at greater risks to develop hypertension, coronary vascular disease, and insulin resistance, and more attention has been paid to the impact of obesity on kidney, recently. ${ }^{1-3}$ Since the first description regarding the relationship between massive obesity and proteinuria in 1974,4 increased evidence demonstrated that obesity-related glomerulopathy (ORG) should be identified as an isolated complication of obesity in adults.1,2 It is, however, unclear whether this condition exists even in children with obesity. ${ }^{3}$

As the prevalence of obesity has been also rising in children in developed countries including Japan, 3,5 this study was conducted to investigate whether obese children have higher risk of renal injury indicating nephritis.

\section{Materials and Methods}

In Japan, the annual urinary screening for renal disease of elementary and junior highschool students has been mandatory since 1974 when the Ministry of Education revised the school health law to identify children at risk of renal failure in adulthood. According to this law, detailed urinalysis is performed for all elementary and junior high-school students living in Japan. The program is described in detail previously. ${ }^{6}$ Briefly, patients positive for protein and/or occult blood on two serial occasions are subjected to a $3^{\text {rd }}$ examination: these patients are asked to attend a medical center for a detailed examination consisting of urinalysis, medical history, physical examination including measurements of weight and height, and laboratory studies, performed by pediatricians. This approach aims to detect any clinical, hematological, or serological abnormalities associated with renal disease. After the $3^{\text {rd }}$ examination, presumptive diagnoses ${ }^{6}$ are made based and grouped into 6 categories as shown in the Table 1.

As a result, eighteen hundred and thirty children aged 6-14 years with abnormal urinary findings detected by the screening program in part of Tokyo in 2007 were enrolled in this study. For comparison of the nutritional status among the categorized subjects, body mass index (BMI) was applied.

For statistical analysis, analysis of variance (ANOVA) and Dunnett's test were used among the 6 groups. P value less than 0.05 was considered significant. All numerical data were expressed as mean and standard deviation.

\section{Results}

Eighteen hundred and thirty subjects were classified into 6 categories (Table 1): 588 in A, 112 in B, 278 in C, 570 in D, 255 in E and 27 in F. As shown in the Table 2, the BMI was significantly higher only in F (nephritis/suspected nephritis) than in groups A (no kidney disease: $\mathrm{P}=0.039$ ).

We further analyzed the difference in blood pressure and serum levels of total cholesterol between the category A and category $\mathrm{F}$ because obesity is known to influence these parameters: significant difference was found in mean systolic blood pressure between the group A and group $\mathrm{F}$ $(\mathrm{P}=0.011)$ in addition to BMI (Table 2).

\section{Discussion}

It is estimated that $7 \%$ of children in the world are obese. 7 The national surveys from the USA found that since 1980 the number of
Correspondence: Kazunari Kaneko, Department of Pediatrics, Kansai Medical University, 2-3-1 Shin-machi, Hirakata-shi, Osaka 573 1191, Japan.

Te1. +81.728 .040 .101 (ext.) 3114

Fax: +81.728 .042 .758$

E-mail:kanekok@hirakata.kmu.ac.jp

Key words: obesity-related glomerulopathy; obesity index; urinary screening; overweight child.

Received for publication: 23 May 2011.

Revision received: 3 October 2011.

Accepted for publication: 26 October 2011.

This work is licensed under a Creative Commons Attribution NonCommercial 3.0 License (CC BYNC 3.0).

(C) Copyright K. Kaneko et al., 2011

Licensee PAGEPress, Italy

Pediatric Reports 2011; 3:e27

doi:10.4081/pr.2011.e27

overweight children has doubled, and the number of overweight adolescents has tripled. ${ }^{8}$ As several lines of evidence demonstrated that ORG should be identified as an isolated complication of obesity in adults, attention has been recently paid to the impact of obesity not only on hypertension, coronary vascular disease, and insulin resistance but also on kidney.1-3 In fact, obese subjects, even in the absence of diabetes, can have glomerulomegaly, thickening of glomerular basement membranes, proliferation of mesangial matrix, focal segmental glomerulosclerosis (FSGS) and increased micoralbuminuria called ORG, both in normotensive and hypertensive subjects. ${ }^{3}$ Kambham et al. brought to our attention a tenfold increase in the prevalence of ORG, 1 from $0.2 \%$ in $1986-1990$ to $2 \%$ in $1996-2000$ in obese subjects (BMI $>30 \mathrm{~kg} / \mathrm{m}^{2}$ ).

These findings prompted us to investigate whether overweight children have an independent risk of developing to kidney disease as well as in adult. As a result, we firstly demonstrated that children having both proteinuria and hematuria are more obese than those without urine abnormalities. This suggests that ORG may develop even in childhood.

In addition to BMI, there was a significant difference only in systolic blood pressure between group $A$ and group $F$, while there were no significant differences among group A, B, $\mathrm{C}, \mathrm{D}$, and group $\mathrm{E}$ as shown in the Table 2 . Recent observations revealed that systolic blood pressure correlates well with BMI than diastolic blood pressure. ${ }^{9}$ Furthermore, a metaanalysis indicated that the change in blood pressure associated with body weight is more pronounced in systolic blood pressure than in diastolic blood pressure. 10 These factors might 
Table 1. Criteria of diagnoses in the urinary screening program in Japan.

No abnormal findings on urinalysis, medical history, physical examination, and laboratory studies

Significant proteinuria ( $>20 \mathrm{mg} / \mathrm{dL}$ ) in the random urine samples but not in the first voided urine sample in the morning Orthostatic proteinuria

Significant proteinuria ( $>20 \mathrm{mg} / \mathrm{dL}$ ) without abnormal findings in medical history, physical examination, and laboratory studies

Significant hematuria (6-20 RBC/HPF) without abnormal findings in medical history, physical examination, and laboratory studies

Significant hematuria (>20 RBC/HPF) without abnormal findings in medical history, physical examination, and laboratory studies

Both proteinuria and hematuria positive cases with or without abnormal findings in medical history, physical examination, and laboratory studies HPF, high power field; RBC, red blood cells.

Table 2. Body mass index, blood pressure and serum cholesterol in the subjects.

\begin{tabular}{lcccccc} 
& Category A & Category B & Category C & Category D & Category E & Category F \\
Number & 588 & 112 & 278 & 570 & 255 & 27 \\
BMI & $18.57 \pm 3.06$ & $18.07 \pm 2.80$ & $18.43 \pm 3.46$ & $17.45 \pm 3.06$ & $17.73 \pm 3.22$ & $20.07 \pm 5.67 *$ \\
\hline Systolic blood pressure (mmHg) & $102.29 \pm 11.81$ & $103.45 \pm 11.31$ & $103.48 \pm 11.92$ & $97.82 \pm 11.73$ & $98.75 \pm 11.19$ & $109.00 \pm 15.56 *$ \\
Diastolic blood pressure (mmHg) & $61.01 \pm 9.83$ & $60.17 \pm 9.18$ & $60.55 \pm 9.18$ & $57.53 \pm 9.67$ & $58.37 \pm 8.71$ & $60.54 \pm 9.00$ \\
\hline Total cholesterol (mg/dL) & $164.61 \pm 26.49$ & $162.66 \pm 28.01$ & $159.50 \pm 23.90$ & $164.21 \pm 27.36$ & $165.14 \pm 26.07$ & $171.00 \pm 26.87$ \\
\hline
\end{tabular}

BMI, body mass index; ${ }^{*} \mathrm{P}<0.05$ compared to group A (children with no kidney diseases: controls).

lead to the finding that there was only significant difference in systolic blood pressure but not in diastolic blood pressure.

The pathogenic mechanism responsible for proteinuria in obese subjects is speculated to be from glomerular hyperfiltration based on direct observation of increased glomerular filtration rate in obese individuals, and indirectly from similarity in clinical course between ORG and renal diseases characterized by glomerular hyperfiltration, such as secondary FSGS.11,12 In addition, recent study revealed that adipose tissue plays a pivotal role in the development of renal injury. Adipose tissue is recognized as a source of inflammatory cytokines such as tumor necrosis factor- $\alpha$ (TNF $\alpha$ ), interleukin-6 (IL-6) and C-reactive protein (CRP) ${ }^{13}$ and CRP is higher in men and women with an elevated body mass index.14 Thus, obesity is associated with a state of lowgrade systemic inflammation, which may contribute to renal injury and cardiovascular disease. In fact, recent observation confirmed this finding demonstrating the increased gene expression of inflammatory cytokines, such as TNF $\alpha$ or IL- 6 RNA in patients with ORG. 15

Taken these findings into consideration, we speculate that intra-glomerular hyperfiltration and inflammatory cytokines play important roles in development of proteinuria and hematuria in obese children, respectively. Another result in the present study that systolic blood pressure is higher in children having proteinuria with hematuria than those without abnormal urinary findings lends support to this speculation because hypertension can be caused by increased inflammatory cytokines, such as TNF $\alpha$ or IL-6.16

There is some limitation in this study: because of the screening-urinalysis based study, patients positive for protein and/or occult blood on three serial occasions are recommended to refer a pediatric nephrologists freely, who make a decision for further examinations including renal biopsy. Therefore, the pathological findings of the children with hematuria and proteinuria cannot be available. The lack of pathological information offers a hindrance to investigate the histopathology of ORG in children.

In conclusion, although the further study is clearly needed, obesity can be associated with the development of renal injury characterized by proteinuria and hematuria even in childhood and early intervention to lose weight should be mandatory, as the benefit of weight loss in ORG in adult is reported to be limited.

\section{References}

1. Kambham N, Markowitz GS, Valeri AM, et al. Obesity-related glomerulopathy: an emerging epidemic. Kidney Int 2001; 59:1498-509.

2. Ross WR, McGill JB. Epidemiology of obesity and chronic kidney disease. Adv Chronic Kidney Dis 2006;13: 325-35.

3. Srivastava T. Nondiabetic consequences of obesity on kidney. Pediatr Nephrol 2006; 21:463-70.

4. Weisinger JR, Kempson RL, Eldridge FL, Swenson RS. The nephrotic syndrome: a complication of massive obesity. Ann Intern Med 1974;81:440-7.

5. Matsushita Y, Yoshiike N, Kaneda F, et al. Trends in childhood obesity in Japan over the last 25 years from the national nutrition survey . Obes Res 2004;12:205-14.

6. Murakami M, Yamamoto H, Ueda Y, et al. Urinary screening of elementary and junior high-school children over a 13-year period in Tokyo. Pediatr Nephrol 1991; 5:50-3.

7. Speiser PW, Rudolf MC, Anhalt H, et al. Childhood obesity. J Clin Endocrinol Metab 2005;90:1871-87.

8. Troiano RP, Flegal KM, Kuczmarski RJ, et al. Overweight prevalence and trends for children and adolescents. The National Health and Nutrition Examination Surveys, 1963 to 1991. Arch Pediatr Adolesc Med 1995;149:1085-91.

9. Gilardini L, Parati G, Sartorio A, et al. Sympathoadrenergic and metabolic factors are involved in ambulatory blood pressure rise in childhood obesity. J Hum Hypertens 2008;22:75-82.

10. Neter JE, Stam BE, Kok FJ, et al. Influence of weight reduction on blood pressure: a meta-analysis of randomized controlled trials. Hypertension 2003;42:878-84.

11. Chagnac A, Weinstein T, Korzets A, et al. Glomerular hemodynamics in severe obe- 
sity. Am J Physiol Renal Physiol 2000; 278:F817-22.

12. Praga M, Morales E, Herrero JC, et al. Absence of hypoalbuminemia despite massive proteinuria in focal segmental glomerulosclerosis secondary to hyperfiltration. Am J Kidney Dis 1999;33:52-8.

13. Hall JE, Crook ED, Jones DW, et al. Mechanisms of obesity-associated cardio- vascular and renal disease. Am J Med Sci 2002;324:127-37.

14. de Jong PE, Verhave JC, Pinto-Sietsma SJ, Hillege HL. PREVEND study group. Obesity and target organ damage: the kidney. Int $\mathrm{J}$ Obes Relat Metab Disord 2002;26 Suppl4:S21-4.

15. Wu Y, Liu Z, Xiang Z, et al. Obesity-related glomerulopathy: insights from gene expression profiles of the glomeruli derived from renal biopsy samples. Endocrinology 2006;147:44-50.

16. Lamarca BD, Ryan MJ, Gilbert JS, et al. Inflammatory Cytokines in the Pathophysiology of Hypertension during Preeclampsia. Curr Hypertens Rep 2007; 9:480-5. 